via their $\mathrm{N}$-terminus or side chain amino group with a 6-aminoor a 6-azidohexanoic acid spacer to provide a convenient attachment site for either 1,4,7,10-tetraazacyclododecane-1,4,7,10-tetraacetic acid (DOTA) or an 2-ethynyl-6-[18F]-fluoropyridine prosthetic group for radiolabelling with ${ }^{68} \mathrm{Ga}$ or ${ }^{18} \mathrm{~F}$, respectively. The $\alpha_{v} \beta_{6}$-binding capability of these peptide derivatives was assessed by competitive binding enzyme-linked immunosorbent assay (ELISA) and flow cytometry. Peptide derivatives that displayed strong affinity for $\alpha_{v} \beta_{6}$ were taken forward to "hot" cell surface binding experiments to evaluate their selectivity for the target. Stability of radiolabelled peptides was measured in human serum.

Competitive binding ELISA experiments (Figure 1) and flow cytometry experiments, showed that cRGD1 and cRGD4 were able to inhibit the binding of $\alpha_{\mathrm{v}} \beta_{6}$-specific 10D5 mAb to $\alpha_{\mathrm{v}} \beta_{6}$ with $\mathrm{IC}_{50}$ values of $6.6 \mathrm{nM}$ and $1.6 \mathrm{nM}$, respectively. Labelling cRGD1 and cRGD4 with fluorine and gallium resulted in $\mathrm{IC}_{50}$ values of $12.2 \mathrm{nM}$ and $1.2 \mathrm{nM}$ for ${ }^{19} \mathrm{~F}$-Pyr-cRGD1 and ${ }^{68} \mathrm{Ga}-$ DOTA-cRGD4, respectively. Serum stability studies of ${ }^{68} \mathrm{Ga}-$ DOTA-cRGD4 have shown that this tracer is $>90 \%$ stable after 2 half-lives of ${ }^{68} \mathrm{Ga}(136 \mathrm{~min})$.

Radiolabelled cyclic RGD peptides have showed favourable binding and stability characteristics to warrant their investigation by PET imaging in vivo. A xenograft murine model using $\alpha_{\mathrm{v}} \beta_{6}{ }^{+}$ and $\alpha_{v} \beta_{6}{ }^{-}$tumours is currently under development to validate tracer uptake and biodistribution in vivo.

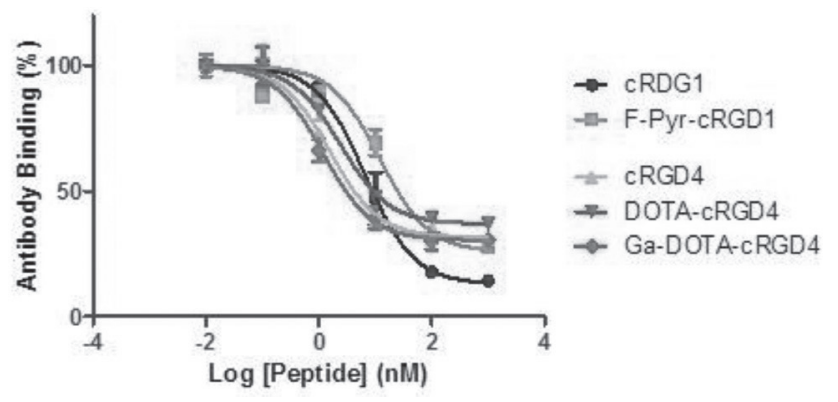

Abstract P40 Figure $1 \alpha_{v} \beta_{\mathrm{e}}$ competitive binding ELISA between labelled/non-labelled cyclic peptides and the $\alpha_{v} \beta_{\mathrm{e}}$-specific mAb (clone10D5)

\section{P41 A PROSPECTIVE COHORT STUDY TO MEASURE IN-VIVO CHANGES IN LUNG GLUCOSE METABOLISM IN PATIENTS WITH SSCL-ILD USING FDG-PET}

HS Garthwaite, V Holmes, T Mikolasch, G Azzopardi, CP Denton, AM Groves, JC Porter. University College London, London, UK

\subsection{6/thoraxjnl-2016-209333.184}

Background Systemic sclerosis (SScl) is a chronic inflammatory autoimmune rheumatic disease with a UK prevalence of $2-10$ per 100,000 . It is a heterogenous disease characterised by varying degrees of dermal and organ fibrosis. Interstitial lung disease (ILD) occurs in $60-80 \%$ of patients and ranges from mild, clinically trivial disease to extensive fibrosis that results in respiratory failure and premature death. Therapeutic options include cyclophosphamide, mycophenylate mofetil and rituximab. Clinical decisions are complex and decisions to treat or not have historically been based on radiology and lung function tests, neither of which (at a single time point) give a dynamic view of disease progression. Novel biomarkers are urgently needed to predict disease activity, progression and response to treatment in patients with SScl-ILD.

Aims To investigate the potential of 18Fluoro-deoxyglucose Positron Emission Tomography (FDG-PET)/CT to act as a prognostic and response biomarker in patients with SScl-ILD.

Methods 35 SScl-ILD patients were prospectively recruited for 18F-FDG-PET/CT. Patients were screened for lung involvement using clinical assessment, chest X-ray and pulmonary function testing (PFT). Those with confirmed SScl-ILD underwent combined high resolution CT scan (HRCT)/PET scanning. The imaging signal and clinical findings were correlated with the need for, and response to, therapy. Follow up was with clinical assessment, PFT and when a change in treatment was indicated, repeat imaging.

Results The overall maximum pulmonary uptake of $18 \mathrm{~F}-\mathrm{FDG}$ (SUVmax), the minimum pulmonary uptake or background-lungactivity (SUVmin) and target-to-background (SUVmax/SUVmin) ratio (TBR) were quantified using routine region-of-interest analysis. Kaplan-Meir analysis was used to identify associations with disease progression and response to treatment

Conclusions We have shown that high pulmonary uptake of $18 \mathrm{~F}$ FDG is associated with disease activity and progression in patients with SScl-ILD. These PET findings can be used to give additional information, supplemental to PFTs, which may then aid clinical treatment decisions.

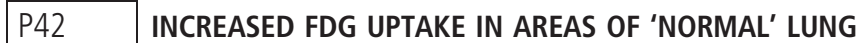 IN IDIOPATHIC PULMONARY FIBROSIS}

${ }^{1} \mathrm{SA}$ Aliyu, ${ }^{2} \mathrm{G}$ Avery, ${ }^{1} \mathrm{AH}$ Morice, ${ }^{1} \mathrm{SP}$ Hart, ${ }^{2} \mathrm{MG}$ Crooks. ${ }^{1}$ University of Hull, Hull, UK; ${ }^{2}$ Hull and East Yorkshire NHS Hospital Trust, Hull, UK

\subsection{6/thoraxjnl-2016-209333.185}

Background Idiopathic pulmonary fibrosis (IPF) has a variable disease course and we lack biomarkers that accurately predict prognosis or treatment response. Positron Emission TomographyComputed Tomography (PET-CT) provides structural and functional information about the lung. A study of 25 IPF patients reported increased $18[\mathrm{~F}]-\mathrm{FDG}$ uptake in areas of normal lung compared to controls. ${ }^{1}$ If confirmed, this raises the possibility that PET-CT can identify 'microscopic fibrosis' with prognostic implications. We assess $18[\mathrm{~F}]-\mathrm{FDG}$ uptake in areas of lung with normal CT appearance in a second IPF cohort.

Methods PET-CT scans undertaken for cancer staging at an interstitial lung disease tertiary referral centre were reviewed. IPF patients and controls without lung disease were identified. 18[F]FDG uptake was assessed using manual region of interest (ROI) placement in areas of lung with normal CT appearance in IPF patients and controls. ROI were placed away from the mediastinum and concomitant tumours. $18[\mathrm{~F}]-\mathrm{FDG}$ uptake within ROI was expressed as maximum and mean standardised uptake values (SUV) normalised using body weight. Mean Hounsfield Units (HU) were evaluated to assess for subtle differences in radiodensity within ROI. Data are presented as mean \pm SD. Unpaired, 2tailed T-tests were used to compare between group differences with a $P$ value $<0.05$ considered significant.

Results Forty-five subjects were included in this study (15 IPF and 30 controls). Lung cancer was the most common concomitant malignancy in both groups.

There was no difference in mean HU within ROI between IPF and controls $(-719 \pm 79 \mathrm{HU}$ in IPF and $-723 \pm 147 \mathrm{HU}$ in 